\title{
EDITORIAL
}

\section{Enterobacteriaceae and Pseudomonas aeruginosa in community-acquired pneumonia: the reality after a decade of uncertainty?}

\author{
A. Torres* and R. Menéndez ${ }^{\#}$
}

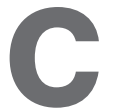
ommunity-acquired pneumonia (CAP) is a frequent infectious respiratory disease that remains a major cause of morbidity and mortality in developed countries. The percentage of CAP patients requiring hospitalisation is not well known and probably varies from country to country, reflecting different criteria for hospitalisation and different resources available. In a very recent epidemiological study of a large series of hospitalised patients with CAP in Germany [1], the overall mortality was $14 \%$, an apparently very high figure. When patients from nursing homes and those chronically bedridden (patients now considered as having healthcare-associated pneumonia (HCAP)) were excluded from the analyses, the overall mortality dropped to $8 \%$, a figure more in line with other recent European studies that excluded nursing-home patients [2].

Mortality in hospitalised CAP patients depends on several factors, including age, comorbidities, microbial aetiology, and early and adequate initial antibiotic treatment. The adequacy of initial antibiotic treatment is the only factor amenable to modification by medical intervention. Initial antibiotic treatment is frequently administered on an empirical basis. Gramnegative Enterobacteriaceae (GNEB) and Pseudomonas aeruginosa are a rare microbial cause of CAP. When these pathogens are involved, mortality increases [3]. Antibiotic treatment for $P$. aeruginosa is completely different from the standard treatment to cover Streptococcus pneumoniae, Legionella pneumophila and atypical pathogens (the most common microorganisms causing CAP). For this reason, most of the current guidelines for CAP include recommendations to suspect and empirically treat $P$. aeruginosa [4].

How frequent is this problem? 10 yrs ago, RuIz et al. [5] reported an incidence of GNEB+Pseudomonas of $9 \%$ in a series of 395 hospitalised patients with CAP. GNEB and P. aeruginosa were associated with pulmonary comorbidities (odds ratio (OR) 3.1). In addition, both microorganisms were associated with the severity of pneumonia (OR 2.5). In a larger series

\footnotetext{
*Pneumology Dept, Clinic Institute of Thorax (ICT), Hospital Clinic of Barcelona, Insitut d'Investigacions Biomèdiques August Pi i Sunyer (IDIBAPS), University of Barcelona (UB), Ciber de Enfermedades Respiratorias (Ciberes), and ${ }^{*}$ Hospital Universitario la Fe de Valencia, Ciber de Enfermedades Respiratorias (Ciberes), Barcelona, Spain.
}

CORRESPONDENCE: A. Torres, Servei de Pneumologia, Hospital Clinic de Barcelona, Villarroel 170 08036 Barcelona, Spain. E-mail: atorres@clinic.ub.es
(559 patients) from our group, $11 \%$ of patients with a microbial diagnosis had GNEB+Pseudomonas [3]. The presence of pulmonary comorbidities (OR 5.8) and previous hospital admission (OR 2.8) were the risk factors associated with these aetiologies. From these and other studies, it appears that the issue of GNEB and $P$. aeruginosa is of importance in hospitalised CAP patients. In addition, mortality and other outcome parameters were clearly worse in these patients.

During the past decade, the concept of HCAP has come to the fore. The St Louis group of MICEK et al. [6] described a group of patients with CAP with very particular risk factors and in whom two important aetiologies were $P$. aeruginosa and methicillin-resistant Staphylococcus aureus. The associated risk factors were transfer from another care facility, receiving longterm haemodialysis, and hospitalisation during the previous 30 days. It is of note that most of this information was collected retrospectively. The idea that HCAP was a different entity from CAP was included in the 2005 American Thoracic Society/Infectious Diseases Society of America guidelines for nosocomial pneumonia [7]. In fact, these guidelines recommended treating patients suffering from HCAP with a combination of antibiotics covering potential resistant microorganisms such as $P$. aeruginosa and methicillin-resistant $S$. aureus. After the release of the guidelines, information from Europe [8] failed to confirm the importance of these microorganisms in patients with HCAP. The current belief is that the concept of HCAP needs to be reconsidered [9] and that, for the purpose of empirical treatment, patients should be stratified into those who can be treated as CAP and those who may benefit from nosocomial pneumonia treatment. The risk factors that may help clinicians to stratify these patients are still not clear and further studies were recommended.

After the release of the 2005 and 2007 guidelines, it was recommended that HCAP patients (particularly nursing-home patients) be excluded from analyses and publications of CAP data. This resulted in a lower frequency of GNEB and $P$. aeruginosa compared with the series from RuIZ et al. [5], published 10 yrs ago. The series of DAMBrava et al. [2], which excluded nursing-home patients, found incidences of GNEB $+P$. aeruginosa of $>1 \%$, figures that support the finding that part of the GNEB and P. aeruginosa aetiologies included in the CAP information of $10 \mathrm{yrs}$ ago probably belonged to HCAP patients. 
In this issue of the European Respiratory Journal, VON BAUM et al. [10], investigators from the German CAP competence network (CAPNETZ), report data based on the analyses of 5,130 hospitalised patients with CAP. The incidence of GNEB and $P$. aeruginosa was $1.3 \%$ (67 cases) and $0.4 \%$ (22 cases), respectively, which are even lower figures than those reported in recent studies on hospitalised CAP patients [11]. Interestingly, 6\% (307) of the patients were nursing-home residents. The inclusion or exclusion of other patients that might meet the definition of HCAP is not reported. The conclusions of the authors are that the incidence of GNEB and P. aeruginosa is very low and, consequently, this is not a major problem in the management of hospitalised CAP patients.

The merits of this study are the large number of patients included and the strict microbiological criteria applied in respiratory samples in order to accept a definite diagnosis. However, in our opinion, the study has some limitations acknowledged in part by the authors. First, a strict protocol to collect samples and respiratory specimens is not reported and was probably not applied to all patients. Accordingly, an unknown percentage of patients with GNEB and P. aeruginosa may have been missed. Secondly, and most important, the authors agree that not all patients admitted to the intensive care unit (ICU) were included. The percentage of patients admitted to the ICU is not reported and the frequency of mechanically ventilated patients was only $2.5 \%$, half the frequency reported in other studies [2]. Given that patients with GNEB and $P$. aeruginosa more frequently require admission to an ICU, it is reasonable to suspect that the authors missed part of this population in their study.

Despite all these considerations, there is no doubt that mortality in CAP is increased several fold when GNEB or $P$. aeruginosa are the microbial aetiologies involved. Knowing the associated risk factors will prompt early suspicion and diagnosis, and adequate initial therapy. In the study that gives rise to this editorial [10], the multivariate analysis found that congestive heart failure and cerebrovascular disease were risk factors for GNEB, while enteral tube feeding and chronic respiratory disease were risk factors for $P$. aeruginosa. Cerebrovascular disease and enteral feeding are risk factors that are more in line with the HCAP concept and they are markers of a population that should be studied separately from $\mathrm{CAP}$. Before any changes in the therapeutic recommendations can be made, we need "clean" studies in CAP and in HCAP hospitalised patients to ascertain which host risk factors increase the probability of acquiring these difficult-to-treat pathogens in both types of patients. Undoubtedly, patients requiring ICU admission must be included for the following reasons: 1) the incidence of GNEB and P. aeruginosa is greater compared with CAP patients outside the ICU, and 2) the effect of inadequate antibiotic treatment on increased mortality is higher compared with other microorganisms. In our opinion, the puzzle of GNEB and P. aeruginosa in CAP and HCAP still has some missing pieces. As suggested above, rigorous studies with a strict microbiological diagnosis work-up and separating CAP and HCAP (which still needs a better definition) will allow us to definitively close the debate.

\section{SUPPORT STATEMENT}

Financial support was received from: 2009 SGR 911 (Barcelona, Spain), Ciber de Enfermedades Respiratorias (Ciberes CB06/06/0028) (Bunyola, Palma de Mallorca, Spain), and el Ciberes es una iniciativa del ISCIII (Madrid, Spain).

\section{STATEMENT OF INTEREST}

A statement of interest for A. Torres can be found at www.erj. ersjournals.com/misc/statements.dtl

\section{REFERENCES}

1 Ewig S, Birkner N, Strauss R, et al. New perspectives on community-acquired pneumonia in 388,406 patients. Results from a nationwide mandatory performance measurement programme in healthcare quality. Thorax 2009; 64: 1062-1069.

2 Dambrava PG, Torres A, Vallès X, et al. Adherence to guidelines' empirical antiobiotic recommendations and community-acquired pneumonia outcome. Eur Respir J 2008; 32: 892-901.

3 Arancibia F, Bauer TT, Ewig S, et al. Community-acquired pneumonia due to Gram-negative bacteria and Pseudomonas aeruginosa: incidence, risk, and prognosis. Arch Intern Med 2002; 162: 1849-1858.

4 Mandell LA, Wunderink RG, Anzueto A, et al. Infectious Diseases Society of America/American Thoracic Society consensus guidelines on the management of community-acquired pneumonia in adults. Clin Infect Dis 2007; 44: Suppl. 2, S27-S72.

5 Ruiz M, Ewig S, Marcos MA, et al. Etiology of communityacquired pneumonia: impact of age, comorbidity, and severity. Am J Respir Crit Care Med 1999; 160: 397-405.

6 Micek ST, Kollef KE, Reichley RM, et al. Health care-associated pneumonia and community-acquired pneumonia: a single-center experience. Antimicrob Agents Chemother 2007; 51: 3568-3573.

7 American Thoracic Society, Infectious Diseases Society of America, Guidelines for the management of adults with hospitalacquired, ventilator-associated, and healthcare-associated pneumonia. Am J Respir Crit Care Med 2005; 171: 388-416.

8 Carratalà J, Mykietiuk A, Fernández-Sabé N, et al. Health careassociated pneumonia requiring hospital admission: epidemiology, antibiotic therapy, and clinical outcomes. Arch Intern Med 2007; 167: 1393-1399.

9 Brito V, Niederman MS. Healthcare-associated pneumonia is a heterogeneous disease, and all patients do not need the same broad-spectrum antibiotic therapy as complex nosocomial pneumonia. Curr Opin Infect Dis 2009; 22: 316-325.

10 von Baum $\mathrm{H}$, Welte $\mathrm{T}$, Marre $\mathrm{R}$, et al. Community-acquired pneumonia through Enterobacteriaceae and Pseudomonas aeruginosa: diagnosis, incidence and predictors. Eur Respir J 2010; 35: 598-605.

11 Falguera M, Carratalà J, Ruiz-González A, et al. Risk factors and outcome of community-acquired pneumonia due to Gramnegative bacilli. Respirology 2009; 14: 105-111. 